\title{
Compactness conditions for elementary operators
}

\author{
by \\ MAtej BrešAr (Maribor) and Yuri V. Turovski (Baku)
}

\begin{abstract}
Various topics concerning compact elementary operators on Banach algebras are studied: their ranges, their coefficients, and the structure of algebras having nontrivial compact elementary operators. In the first part of the paper we consider separately elementary operators of certain simple types. In the second part we obtain our main results which deal with general elementary operators.
\end{abstract}

1. Introduction. Let $\mathcal{A}$ be an algebra. If $\mathcal{A}$ does not have an identity element, then we denote by $\mathcal{A}^{1}$ the algebra obtained from $\mathcal{A}$ by adjoining the identity element (if $\mathcal{A}$ has an identity element, then we set $\mathcal{A}^{1}=\mathcal{A}$ ). Given $a \in \mathcal{A}^{1}$, we define the multiplication operators $L_{a}, R_{a}: \mathcal{A} \rightarrow \mathcal{A}$ by $L_{a}(x)=a x$ and $R_{a}(x)=x a$. An operator $E$ from an algebra $\mathcal{A}$ into itself is called an elementary operator on $\mathcal{A}$ if there exist $a_{i}, b_{i} \in \mathcal{A}^{1}$ such that $E=\sum_{i=1}^{n} L_{a_{i}} R_{b_{i}}$. The elements $a_{i}, b_{i}$ will be called the coefficients of $E$.

In our recent paper [9] compact derivations on Banach algebras were considered. The present paper continues this line of investigations. Our main purpose is to study elementary operators on Banach algebras that are simultaneously compact operators. Occasionally we will also consider elementary operators on general algebras that have a finite rank.

In the first sections $2-6$ we deal with elements $a, b \in \mathcal{A}$ such that some of the operators $L_{a}, R_{a}, L_{a} R_{a}, L_{a}-R_{b}$ are compact or of finite rank. We shall see that these conditions have an impact on the algebraic nature of the algebra in question, so that these local properties (concerning a single element $a$ ) determine the global structure of $\mathcal{A}$. A typical conclusion is that $\mathcal{A}$ contains a central idempotent $e$ such that the ideal $e \mathcal{A}$ is finite-dimensional (i.e. $L_{e}$ has finite rank). The results from these sections have rather short proofs and they vary from elementary observations to somewhat deeper statements (Theorem 3.2, for example).

2000 Mathematics Subject Classification: 47B47, 47B48, 47B07, 46H10.

Key words and phrases: elementary operator, completely finite rank element, completely continuous element, compact element, generalized derivation, operator near-ideal.

The first author was supported by a grant from the Ministry of Science of Slovenia. 
After the first "warm-up" sections we proceed to study general elementary operators (Sections 7-10). Section 7 is devoted to an auxiliary algebraic result which is used as a crucial technical tool in subsequent sections. Section 8 is closely connected to the theory of radicals in Banach algebras developed in [25]. This theory enables us to obtain some information about the range of a compact elementary operator; for instance, it turns out that the spectrum of every element in the range is finite or countable. In Section 9 we show that the existence of a nonzero compact elementary operator on a semisimple Banach algebra $\mathcal{A}$ yields a nice structural property of $\mathcal{A}$ : it has a nonzero socle. Section 10 is concerned with the question whether the coefficients of a compact elementary operator must be compact elements ( $a$ is said to be a compact element if $L_{a} R_{a}$ is a compact operator). This question has been studied before (see e.g. $[13,19,24,26])$. However, we shall use a rather different approach, based on the (purely algebraic) concept of the extended centroid of a prime algebra. The theorem that we obtain generalizes and unifies two results from the literature, the theorem by Fong and Sourour on $\mathcal{B}(X)[13]$ and the theorem by Mathieu on $C^{*}$-algebras [19].

Throughout the paper we will combine analytic and algebraic tools and techniques. Although the main goal of the paper is the study of compactness conditions, in several results we treat algebras over arbitrary fields. Most of the results are later used in the analytic setting, but they might also be of some interest in their own right.

2. Completely finite rank elements. We begin with an introductory algebraic section. We shall gather together several elementary assertions, some of which are perhaps already known. Anyhow, the proofs are short.

We assume throughout this section that $\mathcal{A}$ is an algebra over a field $F$. We shall say that $a \in \mathcal{A}$ is a completely finite rank element if both $L_{a}$ and $R_{a}$ are finite rank operators. In other words, both the right ideal $a \mathcal{A}$ and the left ideal $\mathcal{A} a$ are finite-dimensional. The set of all completely finite rank elements of $\mathcal{A}$ will be denoted by $\mathcal{A}_{\mathrm{cf}}$. It is clear that $\mathcal{A}_{\mathrm{cf}}$ is an ideal of $\mathcal{A}$.

Lemma 2.1. $\mathcal{A}_{\mathrm{cf}}$ is the sum of all finite-dimensional ideals of $\mathcal{A}$. In particular, every element in $\mathcal{A}_{\mathrm{cf}}$ generates a finite-dimensional ideal in $\mathcal{A}$.

Proof. If $a$ lies in some finite-dimensional ideal $\mathcal{J}$ of $\mathcal{A}$, then $a \mathcal{A} \cup \mathcal{A} a \subset \mathcal{J}$ and so $a \in \mathcal{A}_{\mathrm{cf}}$. Therefore it suffices to show that every $a \in \mathcal{A}_{\mathrm{cf}}$ generates a finite-dimensional ideal of $\mathcal{A}$. Since $L_{a}$ is of finite rank, there exists a finite basis $\left\{b_{1}, \ldots, b_{n}\right\}$ of $a \mathcal{A}$. Clearly $R_{b_{1}}, \ldots, R_{b_{n}}$ have finite ranks, and so

$$
\mathcal{I}=F a+L_{a}(\mathcal{A})+R_{a}(\mathcal{A})+\sum_{i=1}^{n} R_{b_{i}}(\mathcal{A})
$$

is finite-dimensional. Note that $\mathcal{I}$ is the ideal of $\mathcal{A}$ generated by $a$. 
By a minimal ideal of an algebra $\mathcal{A}$ we mean of course a nonzero ideal $\mathcal{J}$ of $\mathcal{A}$ such that $0 \subset \mathcal{K} \subset \mathcal{J}$, where $\mathcal{K}$ is an ideal of $\mathcal{A}$, implies $\mathcal{K}=0$ or $\mathcal{K}=\mathcal{J}$, and by a minimal central idempotent in $\mathcal{A}$ we mean a nonzero central idempotent $e$ in $\mathcal{A}$ such that $e f=f$, where $f$ is a central idempotent in $\mathcal{A}$, implies $f=0$ or $f=e$. As usual, by $M_{n}(\mathcal{B})$ we denote the algebra of $n \times n$ matrices over the algebra $\mathcal{B}$.

Lemma 2.2. Let $\mathcal{J}$ be a nonnilpotent finite-dimensional ideal of $\mathcal{A}$.

(i) If $\mathcal{J}$ is minimal, then there is a minimal central idempotent $e$ in $\mathcal{A}$ such that $\mathcal{J}=e \mathcal{A} \cong M_{n}(\mathcal{D})$ for some positive integer $n$ and some finite-dimensional division algebra $\mathcal{D}$.

(ii) If $F$ is a perfect field, then there exists a nonzero idempotent $u \in \mathcal{J}$ and a finite-dimensional nilpotent ideal $\mathcal{N}$ of $\mathcal{A}$ such that $u x-x u$ is in $\mathcal{N}$ for all $x \in \mathcal{A}$.

Proof. We set $\mathcal{N}=\operatorname{rad}(\mathcal{J})$, the (Jacobson) radical of the algebra $\mathcal{J}$. Since $\mathcal{N}=\mathcal{J} \cap \operatorname{rad}(\mathcal{A}), \mathcal{N}$ is an ideal of $\mathcal{A}$. Moreover, as the radical of a finite-dimensional algebra, $\mathcal{N}$ is nilpotent.

Suppose that $\mathcal{J}$ is a minimal ideal. Since $\mathcal{N} \subset \mathcal{J}$ and $\mathcal{J}$ is nonnilpotent, we have $\mathcal{N}=0$. That is, $\mathcal{J}$ is a semisimple algebra. By the Wedderburn structure theorem $\mathcal{J}$ in particular contains an identity element $e$, so that $\mathcal{J}=e \mathcal{A}$ and $e$ is a central idempotent in $\mathcal{A}$. This implies that every ideal of the algebra $\mathcal{J}$ is also an ideal of $\mathcal{A}$. Therefore $\mathcal{J}$ is a simple algebra, and so, again by the Wedderburn theorem, it is isomorphic to $M_{n}(\mathcal{D})$ for some positive integer $n$ and a finite-dimensional division algebra $\mathcal{D}$. If $f$ is a central idempotent in $\mathcal{A}$ such that ef $=f$, then $0 \subset f \mathcal{A} \subset e \mathcal{A}=\mathcal{I}$ and so $f \mathcal{A}=0$ or $f \mathcal{A}=e \mathcal{A}$, which implies $f=0$ or $f=e$. Thus (i) is proved.

To prove (ii), we first apply, using the assumption that $F$ is perfect, the Wedderburn principal theorem (see e.g. [20, Theorem 2.5.37]) to deduce that $\mathcal{J}=\mathcal{B} \oplus \mathcal{N}$ (the vector space direct sum), where $\mathcal{B}$ is a subalgebra of $\mathcal{J}$ isomorphic to $\mathcal{J} / \mathcal{N}$. Since $\mathcal{J}$ is nonnilpotent, $\mathcal{B} \neq 0$. Thus $\mathcal{B}$ is a finite-dimensional semisimple algebra and so in particular it has an identity element $u \neq 0$. For any $y \in \mathcal{J}$ we have $y-u y, y-y u \in \mathcal{N}$. If $x \in \mathcal{A}$ is arbitrary then $u x, x u \in \mathcal{J}$, whence $u x-u x u, x u-u x u \in \mathcal{N}$ and therefore $u x-x u=(u x-u x u)-(x u-u x u) \in \mathcal{N}$.

We shall say that an algebra $\mathcal{A}$ is finitely semiprime if $\mathcal{A}$ has no nonzero finite-dimensional nilpotent ideals. Besides semiprime algebras this class of algebras for instance also includes amenable Banach algebras (see e.g. [15]). Clearly every finite-dimensional minimal ideal $\mathcal{I}$ of a finitely semiprime algebra $\mathcal{A}$ is of the form $\mathcal{J}=e \mathcal{A} \cong M_{n}(\mathcal{D})$.

Corollary 2.3. Let $\mathcal{A}$ be a finitely semiprime algebra with $\mathcal{A}_{\mathrm{cf}} \neq 0$. Then $\mathcal{A}_{\mathrm{cf}}$ is the direct sum of its finite-dimensional minimal ideals. Accord- 
ingly, $\mathcal{A}=\mathcal{A}_{\mathrm{cf}}$ if and only if $\mathcal{A} \cong \bigoplus_{\alpha} M_{n_{\alpha}}\left(\mathcal{D}_{\alpha}\right)$ where $n_{\alpha}$ is a positive integer and $\mathcal{D}_{\alpha}$ is a finite-dimensional division algebra.

Proof. Let $a \in \mathcal{A}_{\mathrm{cf}}$ and let $\mathcal{J}$ be the ideal of $\mathcal{A}$ generated by $a$. By Lemma 2.1, $\mathcal{J}$ is finite-dimensional. Since $\operatorname{rad}(\mathcal{J})$ is a finite-dimensional nilpotent ideal of $\mathcal{A}$ it follows that $\mathcal{J}$ is semisimple. Using the Wedderburn theorem and arguing similarly to the proof of Lemma 2.2 we see that $\mathcal{J}=f \mathcal{A}$ for some central idempotent $f$ in $\mathcal{A}$, and $\mathcal{J}=\mathcal{J}_{1} \oplus \cdots \oplus \mathcal{J}_{n}$ where each $\mathcal{J}_{k}$ is an ideal of $\mathcal{A}$ and a finite-dimensional simple algebra. Clearly, $\mathcal{J}_{k}$ is a finite-dimensional minimal ideal of $\mathcal{A}$. This shows that $\mathcal{A}_{\mathrm{cf}}$ is the sum of its finite-dimensional minimal ideals. We still have to show that this sum is direct. It is clear that $\mathcal{I}_{1} \cap \mathcal{I}_{2}=0$ for any different finite-dimensional minimal ideals $\mathcal{I}_{1}$ and $\mathcal{I}_{2}$. Accordingly, if $\mathcal{I}_{1}=e_{1} \mathcal{A}$ and $\mathcal{I}_{2}=e_{2} \mathcal{A}$ where $e_{1}, e_{2}$ are central idempotents, then $e_{1} e_{2}=0$, and so $e_{1} \mathcal{I}_{2}=0$. From this we infer that a finite-dimensional minimal ideal $\mathcal{I}=e \mathcal{A}$ has trivial intersection with the sum of all finite-dimensional minimal ideals different from $\mathcal{I}$.

The last assertion may be viewed as a slight extension of the classical Wedderburn structure theorem. We remark that if $\mathcal{A}$ is a normed algebra, then the sum in Corollary 2.3 is a topological direct sum.

3. Completely continuous elements. Until further notice we assume that $\mathcal{A}$ is a complex Banach algebra. Let $a \in \mathcal{A}$. We denote by $\sigma(a)$ the spectrum of $a$, and by $r(a)$ its spectral radius. If $\mathcal{A}$ does not have an identity element, then $\sigma(a)$ is taken with respect to $\mathcal{A}^{1}$. As above, by $\operatorname{rad}(\mathcal{A})$ we denote the Jacobson radical of $\mathcal{A}$.

We say that $a$ is a completely continuous element if both $L_{a}$ and $R_{a}$ are compact operators. The set $\mathcal{A}_{\mathrm{cc}}$ of all completely continuous elements in $\mathcal{A}$ is clearly a closed ideal of $\mathcal{A}$, and $\mathcal{A}_{\mathrm{cf}} \subset \mathcal{A}_{\mathrm{cc}}$.

One of motivations for studying completely continuous elements is their connection to compact derivations. If $a \in \mathcal{A}_{\mathrm{cc}}$ then ad $a=L_{a}-R_{a}$ is a compact derivation. Conversely, if $D$ is a compact derivation, then we see from the formulas $L_{D(a)}=\left[D, L_{a}\right]$ and $R_{D(a)}=\left[D, R_{a}\right]$ that the range of $D$ lies in $\mathcal{A}_{\mathrm{cc}}$. This observation was used in our recent paper [9] studying compact derivations, and from the arguments in that paper one can extract some conclusion about completely continuous elements. Theorem 3.2 below, however, is considerably more general. We remark that in particular it yields more refined information concerning compact derivations than given in $[9$, Theorem 2.1].

Suppose that $a \in \mathcal{A}_{\mathrm{cc}}$ is such that $r(a)>0$, i. e. $a$ is not quasinilpotent. Pick a nonzero $\lambda \in \sigma(a)$. Since $L_{a}$ is a compact operator, $\lambda$ is an isolated point in $\sigma(a)$. Therefore there exists a nonzero spectral idempotent $p \in \mathcal{A}^{1}$ of $a$ corresponding to $\lambda$, given by 


$$
p=\frac{1}{2 \pi i} \int_{\Gamma}(\mu-a)^{-1} d \mu,
$$

where $\Gamma$ is an appropriate contour in the complex plane.

Lemma 3.1. Let $a \in \mathcal{A}_{\mathrm{cc}}$ and let $0 \neq \lambda \in \sigma(a)$. If $p$ is a spectral idempotent of a corresponding to $\lambda$, then $p \in \mathcal{A}_{\mathrm{cf}}$. Moreover, $p$ lies in the ideal of $\mathcal{A}$ generated by a.

Proof. Since $L_{(\mu-a)^{-1}}=\left(\mu-L_{a}\right)^{-1}$ for all $\mu$ from the resolvent set of $a, L_{p}$ is a spectral projection of $L_{a}$ corresponding to $\lambda$. Similarly, $R_{p}$ is a spectral projection of $R_{a}$ corresponding to $\lambda$. From the classical theory of compact operators applied to compact operators $L_{a}$ and $R_{a}$ it follows that $L_{p}$ and $R_{p}$ are of finite rank, i.e. $p \in \mathcal{A}_{\mathrm{cf}}^{1}$, and $\left(L_{a}-\lambda\right) L_{p}$ is a quasinilpotent operator on $\mathcal{A}^{1}$. Since $\left(L_{a}-\lambda\right) L_{p}$ is of finite rank, $\left(L_{a}-\lambda\right) L_{p}$ is a nilpotent operator on $\mathcal{A}^{1}$, whence there exists a positive integer $n$ such that $(a p-\lambda p)^{n}=0$. From this we readily infer that $\lambda^{n} p$, and hence also $p$, lies in the ideal of $\mathcal{A}$ generated by a (in particular, $p \in \mathcal{A}$ ).

TheOrem 3.2. Let $\mathcal{A}$ be a Banach algebra and suppose there exists a $\in$ $\mathcal{A}_{\mathrm{cc}} \backslash \operatorname{rad}(\mathcal{A})$. Then there exist a nonzero idempotent $u \in \mathcal{A}_{\mathrm{cf}}$ and a finitedimensional nilpotent ideal $\mathcal{N}$ of $\mathcal{A}$ such that $u x-x u \in \mathcal{N}$ for all $x \in \mathcal{A}$. Moreover, $u$ lies in the ideal of $\mathcal{A}$ generated by a.

Proof. Let $\mathcal{I}$ be the ideal of $\mathcal{A}$ generated by $a$. If $r(a)=0$ then, since $a \notin$ $\operatorname{rad}(\mathcal{A})$, there exists $a^{\prime} \in a \mathcal{A} \subset \mathcal{I}$ with $r\left(a^{\prime}\right)>0$. Therefore we may assume without loss of generality that $r(a)>0$. Now we may use Lemma 3.1 which in particular tells us that there exists a nonzero idempotent $p \in \mathcal{A}_{\mathrm{cf}} \cap \mathcal{I}$. Let $\mathcal{J}$ be the ideal of $\mathcal{A}$ generated by $p$. Note that $\mathcal{J}$ is finite-dimensional by Lemma 2.1, it is not nilpotent since it contains $p$, and $\mathcal{J} \subset \mathcal{I}$. Now Lemma 2.2 (ii) yields the desired conclusion.

Corollary 3.3. Let $\mathcal{A}$ be a Banach algebra such that $\mathcal{A}_{\mathrm{cc}} \backslash \operatorname{rad}(\mathcal{A}) \neq 0$. Then $\mathcal{A}_{\mathrm{cf}} \backslash \operatorname{rad}(\mathcal{A}) \neq 0$, i.e. $\mathcal{A}$ has a nonzero finite-dimensional ideal which is not contained in $\operatorname{rad}(\mathcal{A})$.

The existence of an element in $\mathcal{A}_{\mathrm{cc}} \backslash \operatorname{rad}(\mathcal{A})$ in general does not guarantee that $\mathcal{A}_{\mathrm{cf}}$ contains nonzero central idempotents (just take the algebra of all $n \times n$ complex matrices that have nonzero entries only in the first row).

Corollary 3.4. Let $\mathcal{A}$ be a finitely semiprime Banach algebra and suppose there exists a $\in \mathcal{A}_{\mathrm{cc}} \backslash \operatorname{rad}(\mathcal{A})$. Then there is a nonzero central idempotent $u \in \mathcal{A}_{\mathrm{cf}}$. Moreover, $u$ lies in the ideal of $\mathcal{A}$ generated by $a$.

Our last result in this section will also be derived from Lemma 3.1. Let us point out that $\overline{\mathcal{A}}_{\mathrm{cf}} \subset \mathcal{A}_{\mathrm{cc}}$ are closed ideals of $\mathcal{A}$ (here, $\overline{\mathcal{A}}_{\mathrm{cf}}$ denotes the closure of $\mathcal{A}_{\mathrm{cf}}$ ). 
Corollary 3.5. For any Banach algebra $\mathcal{A}, \mathcal{A}_{\mathrm{cc}} / \overline{\mathcal{A}}_{\mathrm{cf}}$ is a radical Banach algebra. Accordingly, if $\mathcal{A} / \overline{\mathcal{A}}_{\mathrm{cf}}$ is semisimple, then $\overline{\mathcal{A}}_{\mathrm{cf}}=\mathcal{A}_{\mathrm{cc}}$.

Proof. We have to show that $\mathcal{A}_{\mathrm{cc}} / \overline{\mathcal{A}}_{\mathrm{cf}}$ consists of quasinilpotents. Suppose this is not true, that is, there is $a \in \mathcal{A}_{\mathrm{cc}}$ such that $\sigma\left(a+\overline{\mathcal{A}}_{\mathrm{cf}}\right) \neq\{0\}$. Pick $0 \neq \lambda \in \sigma\left(a+\overline{\mathcal{A}}_{\mathrm{cf}}\right)$, and let $f$ be the spectral idempotent of $a+\overline{\mathcal{A}}_{\mathrm{cf}}$ corresponding to $\lambda$. Of course, $\lambda$ also belongs to $\sigma(a)$. Denote by $p$ the spectral idempotent of a corresponding to $\lambda$. Let $\varphi: \mathcal{A}^{1} \rightarrow \mathcal{A}^{1} / \overline{\mathcal{A}}_{\mathrm{cf}}$ be the quotient map. Since $\varphi$ is a unital epimorphism, we have $\varphi\left((\mu-a)^{-1}\right)=(\mu-\varphi(a))^{-1}$ for all $\mu$ from the resolvent set of $a$. Applying $\varphi$ to both sides of (3.1) we see that $\varphi(p)=f$, i.e., $f=p+\overline{\mathcal{A}}_{\mathrm{cf}}$. However, $p \in \mathcal{A}_{\mathrm{cf}}$ by Lemma 3.1 , so that $f=0-$ a contradiction.

Since $\mathcal{A}_{\mathrm{cc}} / \overline{\mathcal{A}}_{\mathrm{cf}}$ is a closed ideal of $\mathcal{A} / \overline{\mathcal{A}}_{\mathrm{cf}}$, the semisimplicity of $\mathcal{A} / \overline{\mathcal{A}}_{\mathrm{cf}}$ of course implies $\overline{\mathcal{A}}_{\mathrm{cf}}=\mathcal{A}_{\mathrm{cc}}$.

4. Left (right) completely continuous elements. We say that $a \in \mathcal{A}$ is a left (resp. right) completely continuous element of $\mathcal{A}$ if $L_{a}$ (resp. $R_{a}$ ) is a compact operator on $\mathcal{A}$. The set of all left (resp. right) completely continuous elements of $\mathcal{A}$ will be denoted by $\mathcal{A}_{\text {lcc }}$ (resp. $\mathcal{A}_{\text {rcc }}$ ). It is clear that $\mathcal{A}_{\text {lcc }}$ and $\mathcal{A}_{\text {rcc }}$ are closed ideals of $\mathcal{A}$, and $\mathcal{A}_{\text {cc }}=\mathcal{A}_{\text {lcc }} \cap \mathcal{A}_{\text {rcc }}$. Further, by $\mathcal{A}_{\text {lcf }}$ (resp. $\mathcal{A}_{\text {rcf }}$ ) we denote the set of all elements $a \in \mathcal{A}$ such that $L_{a}$ (resp. $R_{a}$ ) is of finite rank. Clearly $\mathcal{A}_{\text {lcf }}$ and $\mathcal{A}_{\text {rcf }}$ are ideals of $\mathcal{A}$ and $\mathcal{A}_{\text {cf }}=\mathcal{A}_{\text {lcf }} \cap \mathcal{A}_{\text {rcf }}$. Note also that $\mathcal{A}_{\text {lcf }}$ (resp. $\mathcal{A}_{\text {rcf }}$ ) is the sum of all right (resp. left) finite-dimensional ideals of $\mathcal{A}$.

To avoid a tedious repetition we shall formulate the results in this section only for left completely continuous elements; of course, analogous results can be proved for right completely continuous elements.

Some of the arguments from the previous section still work in the present context. For example, an analogue of Lemma 3.1 is true: If $p$ is a spectral idempotent of $a \in \mathcal{A}_{\mathrm{lcc}}$ corresponding to a nonzero $\lambda \in \sigma(a)$, then $p \in \mathcal{A}_{\mathrm{lcf}}$. This can be used to prove an analogue of Corollary 3.5 , i.e. $\mathcal{A}_{\text {lcc }} / \overline{\mathcal{A}}_{\text {lcf }}$ is a radical Banach algebra. On the other hand, this shows the existence of a nonzero element $p$ in $\mathcal{A}_{\text {lcf }}$. But there is another, simpler way to establish that $\mathcal{A}_{\text {lcf }} \neq\{0\}$, which we will now show.

We say that a nonzero $b \in \mathcal{A}$ is a left eigenvector of $a \in \mathcal{A}$ corresponding to an eigenvalue $\lambda \in \mathbb{C}$ if $(a-\lambda) b=0$. Note that every $a \in \mathcal{A}_{\text {lcc }}$ with $r(a)>0$ has nonzero eigenvalues.

Lemma 4.1. Let $\mathcal{A}$ be a Banach algebra, and let $a \in \mathcal{A}_{\mathrm{lcc}}$. If $b$ is a left eigenvector of a corresponding to a nonzero eigenvalue, then $b \in \mathcal{A}_{\mathrm{lcf}}$. Moreover, if $\mathcal{A}$ is semiprime, then $b \in \mathcal{A}_{\mathrm{cf}}$.

Proof. Let $(a-\lambda) b=0$ with a nonzero $\lambda \in \mathbb{C}$. Then $\left(L_{a}-\lambda\right)(b \mathcal{A})=0$. Since $L_{a}$ is compact, the eigenspace $\operatorname{ker}\left(L_{a}-\lambda\right)$ is finite-dimensional and so 
$b \mathcal{A}$ must also be finite-dimensional, that is, $b \in \mathcal{A}_{\text {lcf }}$. If $\mathcal{A}$ is semiprime, then Smyth's lemma [23] tells us that $\operatorname{dim}(\mathcal{A} b)<\infty$ as well, so that $b \in \mathcal{A}_{\mathrm{cf}}$.

Is is easy to find an example of a (nonsemiprime) algebra $\mathcal{A}$ containing an idempotent $e$ such that, $\operatorname{say}, \operatorname{dim}(e \mathcal{A})<\infty$ but $\operatorname{dim}(\mathcal{A} e)=\infty$. This justifies the assumption that $\mathcal{A}$ is semiprime in the last assertion. If, however, we impose the stronger assumption on $a$ that $a \in \mathcal{A}_{\mathrm{cc}}$, then the semiprimeness is redundant. Namely, as above we have $\operatorname{dim}(b \mathcal{A})<\infty$, while for the proof of $\operatorname{dim}(\mathcal{A} b)<\infty$ we can make use of dual operators: noting that $\left(R_{a}^{*}-\lambda\right) R_{b}^{*}=0$ with $R_{a}^{*}$ compact, it follows by the same argument as above that $R_{b}^{*}$, and hence also $R_{b}$, is of finite rank.

Corollary 4.2. Let $\mathcal{A}$ be a Banach algebra such that $\mathcal{A}_{\mathrm{lcc}} \backslash \operatorname{rad}(\mathcal{A}) \neq 0$. Then $\mathcal{A}_{\text {lcf }} \neq 0$. Moreover, if $\mathcal{A}$ is semiprime, then $\mathcal{A}_{\mathrm{cf}}$ contains a nonzero central idempotent.

Proof. As in the proof of Theorem 3.2 we see that there is $a \in \mathcal{A}_{\text {lcc }}$ with $r(a)>0$. Hence $a$ has a nonzero eigenvalue, and by Lemma 4.1 every corresponding eigenvector lies in $\mathcal{A}_{\mathrm{lcf}}$. If $\mathcal{A}$ is semiprime, then it lies in $\mathcal{A}_{\mathrm{cf}}$, and then Corollary 2.3 and Lemma 2.2(i) yield the desired conclusion.

Prime algebras clearly do not contain central idempotents different from 0 and 1 . The conclusion is therefore more definite in this case.

Corollary 4.3. Let $\mathcal{A}$ be a prime Banach algebra. If $\mathcal{A}_{\mathrm{lcc}} \backslash \operatorname{rad}(\mathcal{A}) \neq 0$, then $\operatorname{dim}(\mathcal{A})<\infty$.

5. Compact elements. We say that $a \in \mathcal{A}$ is a compact element if $L_{a} R_{a}$ is a compact operator. The set $\mathcal{A}_{\mathrm{c}}$ of all compact elements in $\mathcal{A}$ is only a closed multiplicative semigroup in $\mathcal{A}$ in general.

Finally, we say that $a$ is a finite rank element if $L_{a} R_{a}$ is a finite rank operator. The set of all finite rank elements in $\mathcal{A}$ will be denoted by $\mathcal{A}_{\mathrm{f}}$. Of course, $\mathcal{A}_{\mathrm{f}} \subset \mathcal{A}_{\mathrm{c}}$.

Our intention is to establish results on compact elements that are analogous to those from the previous sections. However, all the necessary work has basically already been done before, so we will just refer to the literature and the present section will be very short. The lemma below follows immediately from a slightly more general result of [21, Lemma 3].

Lemma 5.1 ([21]). Let $\mathcal{A}$ be a Banach algebra. If $\mathcal{A}_{\mathrm{c}} \backslash \operatorname{rad}(\mathcal{A}) \neq 0$, then $\mathcal{A}_{\mathrm{f}}$ contains a nonzero idempotent. In particular, $\mathcal{A}_{\mathrm{f}} \backslash \operatorname{rad}(\mathcal{A}) \neq 0$.

In the case when $\mathcal{A}$ is a semiprime Banach algebra, $\mathcal{A}_{\mathrm{f}}$ coincides with $\operatorname{soc}(\mathcal{A})$, the socle of $\mathcal{A}$. For semisimple algebras this was proved a long time ago [1] and somewhat more recently it was extended to the semiprime case [10]. Moreover, algebraic versions of this result were obtained even more 
recently $[7,14]$ (more details about [7] are given in Section 9). Recall that the socle of a semiprime algebra $\mathcal{A}$ is equal to the sum of all minimal left (resp. right) ideals each of which is necessarily of the form $\mathcal{A} e$ (resp. e $\mathcal{A}$ ) where $e$ is a minimal idempotent in $\mathcal{A}$, i.e. an idempotent such that $e \mathcal{A} e$ is a division algebra. In the case when $\mathcal{A}$ is a Banach algebra we necessarily have $e \mathcal{A} e=\mathbb{C} e$. If $\mathcal{A}$ has no nonzero minimal left (or equivalently, right) ideals then we define $\operatorname{soc}(\mathcal{A})=0$.

Corollary 5.2. Let $\mathcal{A}$ be a semiprime Banach algebra. Then $\mathcal{A}_{\mathrm{c}} \backslash$ $\operatorname{rad}(\mathcal{A}) \neq 0$ if and only if $\operatorname{soc}(\mathcal{A}) \neq 0$.

6. Compact generalized derivations. Following [6] we say that a linear map $\Delta: \mathcal{A} \rightarrow \mathcal{A}$ is a generalized derivation if there exists a derivation $D$ on $\mathcal{A}$ such that $\Delta(x y)=\Delta(x) y+x D(y)$ for all $x, y \in \mathcal{A}$. Besides derivations, the other basic examples are inner generalized derivations, that is, maps of the form $\Delta=L_{a}-R_{b}$ for some $a, b \in \mathcal{A}$ (in this case the associated derivation is $D=\operatorname{ad} b$ ).

In Section 3 we mentioned that completely continuous elements naturally appear when studying compact derivations. Similarly the "one-sided" completely continuous elements appear when studying compact generalized derivations. If $\Delta$ is a compact generalized derivation, then we see from $R_{D(a)}=\left[\Delta, R_{a}\right]$ that $D(a) \in \mathcal{A}_{\text {rcc }}$ for every $a \in \mathcal{A}$. The next result therefore follows from (the "right" version of) Corollary 4.2.

COROllary 6.1. Let $\Delta$ be a compact generalized derivation on a Banach algebra $\mathcal{A}$. Suppose that the associated derivation $D$ does not map $\mathcal{A}$ into $\operatorname{rad}(\mathcal{A})$. Then $\mathcal{A}_{\mathrm{rcf}} \neq 0$; moreover, if $\mathcal{A}$ is semiprime, then $\mathcal{A}_{\mathrm{cf}}$ contains a nonzero central idempotent.

If $\Delta=L_{a}-R_{b}$ is an inner generalized derivation, then Corollary 6.1 says that if $\Delta$ is compact and $b$ does not lie in the center modulo the Jacobson radical, then $\mathcal{A}_{\text {rcf }} \neq 0$. Of course, similarly one can prove that if $\Delta$ is compact and $a$ does not lie in the center modulo the Jacobson radical, then $\mathcal{A}_{\mathrm{lcf}} \neq 0$.

In the semisimple case the above result gets a simple form.

Corollary 6.2. Let $\mathcal{A}$ be a semisimple Banach algebra. If there exists a nonzero compact generalized derivation on $\mathcal{A}$, then $\mathcal{A}_{\mathrm{cf}}$ contains a nonzero central idempotent.

Proof. If the associated derivation $D$ is not zero, then we may use Corollary 6.1. So we may assume that $D=0$. Note that then $\Delta L_{a}=L_{\Delta(a)}$ for every $a \in \mathcal{A}$, and so the result follows from Corollary 4.2.

Corollary 6.2 was obtained for ordinary derivations in [9, Corollary 2.6].

There exists a commutative prime radical Banach algebra $\mathcal{A}$ such that $\mathcal{A}_{\mathrm{lcc}}=\mathcal{A}$ (see [11]). Thus, every $L_{a}\left(=R_{a}\right), a \in \mathcal{A}$, is compact and 
quasinilpotent. Since $\mathcal{A}$ is prime, $\mathcal{A}_{\mathrm{lcf}}=\mathcal{A}_{\mathrm{cf}}=0$, and therefore, because of commutativity of $\mathcal{A}$, even $\mathcal{A}_{\mathrm{f}}=0$. In light of this example, the next proposition seems to be of some interest.

Proposition 6.3. Let $\mathcal{A}$ be a Banach algebra. If there exists a compact inner generalized derivation on $\mathcal{A}$ which is not quasinilpotent, then $\mathcal{A}_{\mathrm{f}} \neq 0$.

Proof. Let $\Delta=L_{a}-R_{b}$ be compact and nonquasinilpotent. Pick a nonzero $\lambda \in \sigma(\Delta)$. Then $X=\operatorname{ker}(\Delta-\lambda)$ is a finite-dimensional space which is invariant under $L_{a}$. Therefore there exists a nonzero element $x \in X$ such that $a x=\mu x$ for some $\mu \in \mathbb{C}$, which in turn implies $x b=(\mu-\lambda) x$. Accordingly,

$$
\Delta L_{x} R_{x}=\lambda L_{x} R_{x} .
$$

Take a spectral projection $P$ of $\Delta$ such that $1-P$ has finite rank and $r(\Delta P)<|\lambda|$. Then

$$
(\Delta P) P L_{x} R_{x}=\lambda P L_{x} R_{x},
$$

whence $P L_{x} R_{x}=0$ and $L_{x} R_{x}=(1-P) L_{x} R_{x}$ is of finite rank.

7. On operator near-ideals. This section is entirely algebraic. By $\mathcal{A}$ we denote an algebra over an arbitrary field. Let $\mathcal{U}$ be a subspace of the algebra of linear operators on $\mathcal{A}$. We shall say that $\mathcal{U}$ is an operator near-ideal on $\mathcal{A}$ if $E U, U E \in \mathcal{U}$ for every $U \in \mathcal{U}$ and every elementary operator $E$ on $\mathcal{A}$. Basic examples of operator near-ideals are of course ideals of the algebra of linear operators on $\mathcal{A}$ (or bounded linear operators in the case when $\mathcal{A}$ is a Banach algebra). Further, we shall say that $a \in \mathcal{A}$ is a $\mathcal{U}$-element if the elementary operator $L_{a} R_{a}$ belongs to $\mathcal{U}$. For example, if $\mathcal{U}$ is the space of all finite rank operators on an algebra $\mathcal{A}$, then by the very definition $a$ is a $\mathcal{U}$-element if and only if $a \in \mathcal{A}_{\mathrm{f}}$. Similarly, if $\mathcal{U}$ is the space of all compact operators on a Banach algebra $\mathcal{A}$, then $a$ is a $\mathcal{U}$-element if and only if $a \in \mathcal{A}_{\mathrm{c}}$.

We shall say that an elementary operator $E$ has length $n$ if $E=$ $\sum_{i=1}^{n} L_{a_{i}} R_{b_{i}}$ for some $a_{i}, b_{i} \in \mathcal{A}^{1}$ and $E$ cannot be represented as $\sum_{i=1}^{k} L_{c_{i}} R_{d_{i}}$ for some $k<n$ and $c_{i}, d_{i} \in \mathcal{A}^{1}$. We also define that the operator 0 has length 0 .

Lemma 7.1. Let $\mathcal{A}$ be an arbitrary algebra and let $\mathcal{U}$ be an operator nearideal on $\mathcal{A}$. If $\mathcal{U}$ contains a nonzero elementary operator, then $\mathcal{A}$ contains a nonzero $\mathcal{U}$-element.

Proof. Let $E=\sum_{i=1}^{n} L_{a_{i}} R_{b_{i}}$ be a nonzero elementary operator of length $n \geq 1$ belonging to $\mathcal{U}$. We proceed by induction on $n$.

Let $n=1$, i.e. $E=L_{a_{1}} R_{b_{1}} \in \mathcal{U}$ and $E \neq 0$. Therefore there exists $t \in \mathcal{A}$ such that $a=a_{1} t b_{1}=L_{a_{1}} R_{b_{1}}(t) \neq 0$. Note that $L_{a} R_{a}=E L_{t b_{1}} R_{a_{1} t}$ belongs to $\mathcal{U}$ and so the assertion is proved in this case. 
We may now assume that $n>1$ and that the assertion is true whenever $\mathcal{U}$ contains a nonzero elementary operator of length $<n$. Pick $y \in \mathcal{A}$ and define $F=E R_{b_{n} y}-R_{y b_{n}} E \in \mathcal{U}$. Note that

$$
F=\sum_{i=1}^{n-1} L_{a_{i}} R_{b_{n} y b_{i}-b_{i} y b_{n}}
$$

and so the length of $F$ is $<n$. In view of the induction assumption we may therefore assume without loss of generality that $F=0$. That is,

$$
\sum_{i=1}^{n-1} L_{a_{i}} R_{b_{n} y b_{i}}=\sum_{i=1}^{n-1} L_{a_{i}} R_{b_{i} y b_{n}}
$$

for every $y \in \mathcal{A}$. Accordingly,

$$
\begin{aligned}
L_{E(x)} R_{b_{n}}(y) & =E(x) y b_{n}=\sum_{i=1}^{n} a_{i} x b_{i} y b_{n}=\sum_{i=1}^{n} L_{a_{i}} R_{b_{i} y b_{n}}(x) \\
& =\sum_{i=1}^{n} L_{a_{i}} R_{b_{n} y b_{i}}(x)=\sum_{i=1}^{n} a_{i} x b_{n} y b_{i}=E L_{x b_{n}}(y)
\end{aligned}
$$

for all $x, y \in \mathcal{A}$, that is,

$$
L_{E(x)} R_{b_{n}}=E L_{x b_{n}}
$$

for all $x \in \mathcal{A}$. Thus $\mathcal{U}$ contains elementary operators $L_{E(x)} R_{b_{n}}, x \in \mathcal{A}$, of length $\leq 1$. If for some $x \in \mathcal{A}$ the length of $L_{E(x)} R_{b_{n}}$ is 1 then the result follows from the induction assumption. Therefore we may assume that $L_{E(x)} R_{b_{n}}=0$ for all $x \in \mathcal{A}$. In fact, the same argument shows that we may assume that for every $j, 1 \leq j \leq n$, we have $L_{E(x)} R_{b_{j}}=0$ for all $x \in \mathcal{A}$. Consequently, $L_{E(x)} R_{a_{j} x b_{j}}=0$ and hence also $L_{E(x)} R_{E(x)}=0$ for all $x \in \mathcal{A}$. In particular, $E(x)$ is a $\mathcal{U}$-element. By assumption $E(x) \neq 0$ for some $x \in \mathcal{A}$ and so the desired conclusion holds true in this case as well.

8. On the range of a compact elementary operator. We are now ready to tackle general elementary operators that are compact. In this section we are interested in the range of such an operator. First we have to recall some notions and results from the theory of radicals of Banach algebras.

A hereditary topological radical on the class of all Banach algebras is a map $\mathcal{A} \mapsto \mathcal{A}_{r}$ which assigns to each Banach algebra $\mathcal{A}$ its closed ideal $\mathcal{A}_{r}$ so that the following conditions are satisfied:

(r) $\left(\mathcal{A} / \mathcal{A}_{r}\right)_{r}=0$.

(rr) $\mathcal{J}_{r}=\mathcal{J} \cap \mathcal{A}_{r}$ for every closed ideal $\mathcal{J}$ of $\mathcal{A}$.

(rrr) $f\left(\mathcal{A}_{r}\right) \subset \mathcal{B}_{r}$ for every continuous epimorphism $f: \mathcal{A} \rightarrow \mathcal{B}$.

The following definitions are taken from [25]. A Banach algebra $\mathcal{A}$ is called hypocompact if a nonzero quotient of $\mathcal{A}$ by an arbitrary closed ideal 
always contains a nonzero compact element, and is called scattered if the spectrum of every element $a \in \mathcal{A}$ is finite or countable. A closed ideal $\mathcal{J}$ of $\mathcal{A}$ is called hypocompact (resp. scattered) if $\mathcal{J}$ is hypocompact (resp. scattered) as a Banach algebra. The next result, which is of crucial importance for our results in this section, follows from [25, Theorems 4 and 6] (see also [22]).

TheOREm 8.1 ([25]). For every Banach algebra $\mathcal{A}$ there exist the largest hypocompact ideal $\mathcal{A}_{\mathrm{hc}}$ and the largest scattered ideal $\mathcal{A}_{\mathrm{sc}}$, and $\mathcal{A}_{\mathrm{hc}} \subset \mathcal{A}_{\mathrm{sc}}$. The maps $\mathcal{A} \mapsto \mathcal{A}_{\mathrm{hc}}$ and $\mathcal{A} \mapsto \mathcal{A}_{\mathrm{sc}}$ are hereditary topological radicals.

Let us add a few elementary remarks.

Lemma 8.2. If $\mathcal{A}$ is a Banach algebra then $\mathcal{A}_{\mathrm{c}} \subset \mathcal{A}_{\mathrm{hc}}$.

Proof. Let $a \in \mathcal{A}_{\mathrm{c}}$ and let $\mathcal{I}$ be the closed ideal of $\mathcal{A}$ generated by $a$. If $\mathcal{J}$ is a proper closed ideal of $\mathcal{I}$, then $a \notin \mathcal{J}$ and so $a+\mathcal{J} \neq 0$. But $a+\mathcal{J}$ is a compact element of $\mathcal{I} / \mathcal{J}$. This shows that $\mathcal{I}$ is hypocompact, and so $a \in \mathcal{I} \subset \mathcal{A}_{\mathrm{hc}}$.

Therefore $\mathcal{A}_{\text {hc }}$ contains the closed ideal of $\mathcal{A}$ generated by $\mathcal{A}_{\mathrm{c}}$. In general $\mathcal{A}_{\text {hc }}$ does not coincide with this ideal.

So we now know that, for every Banach algebra $\mathcal{A}$, we have

$$
\mathcal{A}_{\mathrm{f}} \subset \mathcal{A}_{\mathrm{c}} \subset \mathcal{A}_{\mathrm{hc}} \subset \mathcal{A}_{\mathrm{sc}} \text {. }
$$

If $\mathcal{A}$ is semiprime, and in particular if it is semisimple, then $\mathcal{A}_{\mathrm{f}}=\operatorname{soc}(\mathcal{A})$ (cf. Section 5).

Lemma 8.3. If $\mathcal{A}$ is a semisimple Banach algebra, then $\mathcal{A}_{\mathrm{sc}} \neq 0$ if and only if $\operatorname{soc}(\mathcal{A}) \neq 0$.

Proof. In view of (8.1) we only have to prove the "only if" part. So let $\mathcal{A}_{\mathrm{sc}} \neq 0$. As an ideal of a semisimple algebra, $\mathcal{A}_{\mathrm{sc}}$ is also semisimple. Therefore $\operatorname{soc}\left(\mathcal{A}_{\mathrm{sc}}\right) \neq 0$ by Barnes' theorem [4, Theorem 2.2]. This means that there exists a minimal idempotent $e$ of $\mathcal{A}_{\mathrm{sc}}$. Note that

$$
e \mathcal{A}_{\mathrm{sc}} e \subset e \mathcal{A} e=e(\mathcal{A} e) e \subset e \mathcal{A}_{\mathrm{sc}} e,
$$

whence $e \mathcal{A} e=e \mathcal{A}_{\mathrm{sc}} e=\mathbb{C} e$, i.e. $e$ is a minimal idempotent of $\mathcal{A}$. Therefore $\operatorname{soc}(\mathcal{A}) \neq 0$.

Having Lemma 7.1 in hand it is now easy to connect the theory just sketched with compact elementary operators.

THEOREM 8.4. If $E$ is a compact elementary operator on a Banach algebra $\mathcal{A}$, then the range of $E$ lies in $\mathcal{A}_{\mathrm{hc}}$. Accordingly, $\sigma(E(y) x)$ is finite or countable for all $x, y \in \mathcal{A}^{1}$.

Proof. Elementary operators clearly leave each ideal invariant. In particular, $\mathcal{A}_{\mathrm{hc}}$ is invariant under $E$. Let $E^{\prime}$ be the operator on $\mathcal{A} / \mathcal{A}_{\mathrm{hc}}$ induced by $E$. Obviously, $E^{\prime}$ is also a compact elementary operator. If $E^{\prime}$ 
were nonzero then $\mathcal{A} / \mathcal{A}_{\text {hc }}$ would contain a nonzero compact element by Lemma 7.1. However, in view of the property $(r)$ and Lemma 8.2 this is impossible. Therefore $E^{\prime}=0$, that is, $E$ maps $\mathcal{A}$ into $\mathcal{A}_{\mathrm{hc}}$. By Theorem 8.1 the range of $E$ lies also in $\mathcal{A}_{\mathrm{sc}}$. Taking $\mathcal{A}^{1}$ instead of $\mathcal{A}$ and repeating the argument, we infer that the range of $E$ lies in $\mathcal{A}_{\mathrm{sc}}^{1}$. Hence $\sigma(E(y) x)$ is finite or countable for every $x, y \in \mathcal{A}^{1}$.

COROLlaRY 8.5. If $\sum_{i=1}^{n} L_{a_{i}} R_{b_{i}}$ is a compact operator, then $\sigma\left(\sum_{i=1}^{n} a_{i} b_{i}\right)$ is finite or countable.

9. On algebras having compact elementary operators. Let $\mathcal{A}$ be a semiprime algebra over a field $F$. The sum of all minimal left ideals $e \mathcal{A}$, where $e$ is a minimal idempotent in $\mathcal{A}$ such that the division algebra $e \mathcal{A} e$ is finite-dimensional over $F$, is called the lower socle of $\mathcal{A}$ and will be denoted by $\operatorname{soc}(\mathcal{A})$ (in the Banach algebra case we of course have $\underline{\operatorname{soc}}(\mathcal{A})=\operatorname{soc}(\mathcal{A})$ ). This concept was introduced and studied in the recent paper [7]. It is easy to see that $\operatorname{soc}(\mathcal{A})$ is an ideal of $\mathcal{A}$ (contained of course in $\operatorname{soc}(\mathcal{A})$ ). The important result for us is [7, Theorem 3.3] stating that $\operatorname{soc}(\mathcal{A})=\mathcal{A}_{\mathrm{f}}$. So in particular $\mathcal{A}_{\mathrm{f}}$ is an ideal of $\mathcal{A}$ (this is not clear from the definition). We can now easily prove the following theorem that gives an additional insight into the topic considered in [7, Section 4].

TheOREM 9.1. Let $\mathcal{A}$ be a semiprime algebra. If there exists a nonzero finite rank elementary operator on $\mathcal{A}$, then $\underline{\operatorname{soc}}(\mathcal{A}) \neq 0$.

Proof. Using Lemma 7.1 with $\mathcal{U}$ being the space of all finite rank operators on $\mathcal{A}$ we see that $\mathcal{A}$ contains a nonzero $\mathcal{U}$-element, that is to say, $\underline{\operatorname{soc}}(\mathcal{A})=\mathcal{A}_{\mathrm{f}} \neq 0$.

In a similar fashion we obtain an analytic version of Theorem 9.1.

TheOREM 9.2. Let $\mathcal{A}$ be a semisimple Banach algebra. If there exists a nonzero compact elementary operator on $\mathcal{A}$, then $\operatorname{soc}(\mathcal{A}) \neq 0$.

Proof. We now apply Lemma 7.1 for the case where $\mathcal{U}$ is the space of compact linear operators on $\mathcal{A}$. Hence it follows that $\mathcal{A}$ has a nonzero compact element, and so Corollary 5.2 gives $\operatorname{soc}(\mathcal{A}) \neq 0$.

Note that Theorem 9.2 also follows from Theorem 8.1, Lemma 8.3 and Theorem 8.4.

There are no nonzero compact elementary operators on the Calkin algebra of operators on a separable Hilbert space. This result was conjectured in [13] and proved in [2] (and later also in [16] and [19]). The following corollary is its generalization. 
Corollary 9.3. Let $\mathcal{A}$ be a simple unital Banach algebra. If there exists a nonzero compact elementary operator on $\mathcal{A}$, then $\mathcal{A} \cong M_{n}(\mathbb{C})$ for some $n \geq 1$.

Proof. By Theorem 9.2 we have $\operatorname{soc}(\mathcal{A}) \neq 0$. Since $\mathcal{A}$ is simple and unital it follows that $1 \in \operatorname{soc}(\mathcal{A})=\mathcal{A}_{\mathrm{f}}$. Thus $\mathcal{A}=L_{1} R_{1}(\mathcal{A})$ is finite-dimensional, and hence $\mathcal{A} \cong M_{n}(\mathbb{C})$.

We shall say that a Banach algebra is bicompact if $L_{a} R_{b}$ is a compact operator for all $a, b \in \mathcal{A}$. This is of course a generalization of the concept of a compact Banach algebra [1].

Proposition 9.4. Let $\mathcal{A}$ be a topologically simple Banach algebra. If there exists a nonzero compact elementary operator on $\mathcal{A}$, then $\mathcal{A}$ is a bicompact algebra.

Proof. By Lemma 7.1, $\mathcal{A}$ contains a nonzero compact element $a$. Note that $L_{x a y} R_{z a w}=L_{x} R_{w}\left(L_{a} R_{a}\right) L_{y} R_{z}$ is compact for all $x, y, z, w \in \mathcal{A}$. Consequently, $L_{u} R_{v}$ is compact for all $u, v$ from the ideal of $\mathcal{A}$ generated by $a$. Since this ideal is dense in $\mathcal{A}$, it follows that $\mathcal{A}$ is a bicompact algebra.

If we add to Proposition 9.4 the further assumption that $\mathcal{A}$ is semisimple, then it follows from Theorem 9.2 that $\mathcal{A}$ is equal to the closure of $\operatorname{soc}(\mathcal{A})$; note that this conclusion is stronger than the one given in the proposition. However, it is not known whether a topologically simple Banach algebra is automatically semisimple, i.e. whether there exists a topologically simple radical Banach algebra.

10. On the coefficients of a compact elementary operator. If the algebra $\mathcal{A}$ is not prime then $L_{a} R_{b}$ can be zero although both $a$ and $b$ are nonzero. Therefore it does not seem to be easy to get some interesting information about the coefficients of an elementary operator in nonprime algebras. We shall therefore confine ourselves to prime algebras.

Lemma 7.1 will be indirectly used also in this section (via Theorem 9.2). But we also need other tools; a disadvantage of the approach based on this lemma is that we lose track of the coefficients of an elementary operator in question. We shall now present an alternative approach based on an algebraic result by Erickson, Martindale and Osborn [12, Theorem 3.1] (see also generalizations in [5, Theorem 2.3.3] and [8, Theorem 1.2]) which is stated (in an equivalent form) below as Theorem 10.1. This result involves the concept of the extended centroid of a prime algebra and we refer the reader to the book [5] for an account of this theory. Let us just mention here that the extended centroid of a prime algebra $\mathcal{A}$ over a field $F$ is a certain field containing $F$ (and in fact also containing the center of $\mathcal{A}$ ). In the case when the extended centroid coincides with $F$ we say that $\mathcal{A}$ 
is centrally closed over $F$. By a centrally closed prime Banach algebra we shall of course mean a prime Banach algebra that is centrally closed over $\mathbb{C}$. For example, primitive Banach algebras are centrally closed prime Banach algebras (this follows easily from [5, Corollary 4.1.2]). On the other hand, commutative prime algebras (i.e. commutative domains) of dimension more than 1 over a field $F$ are not centrally closed over $F$ and for such algebras the conclusion of the next theorem clearly does not hold.

TheOREm 10.1 ([12]). Let $\mathcal{A}$ be a centrally closed prime algebra over a field $F$. If $b_{1}, b_{2}, \ldots, b_{n} \in \mathcal{A}^{1}$ are such that $b_{1}$ does not lie in the linear span of $b_{2}, \ldots, b_{n}$, then there exists an elementary operator $E$ on $\mathcal{A}$ such that $E\left(b_{1}\right) \neq 0$ and $E\left(b_{2}\right)=\cdots=E\left(b_{n}\right)=0$.

Let us record a simple corollary to this theorem which indicates both the similarities and differences between the present approach and the one based on Lemma 7.1.

COROLlary 10.2. Let $\mathcal{A}$ be a centrally closed prime algebra over a field $F$ and let $E=\sum_{i=1}^{n} L_{a_{i}} R_{b_{i}}$ be an elementary operator on $\mathcal{A}$ such that $b_{1}$ does not lie in the linear span of $b_{2}, \ldots, b_{n}$. If $E$ belongs to an operator near-ideal $\mathcal{U}$ on $\mathcal{A}$, then there is a nonzero $b \in \mathcal{A}$ such that $L_{a_{1}} R_{b} \in \mathcal{U}$.

Proof. By Theorem 10.1 there is an elementary operator $G=\sum_{j=1}^{m} L_{c_{j}} R_{d_{j}}$ such that $G\left(b_{1}\right)=b \neq 0$ and $G\left(b_{i}\right)=0, i=2, \ldots, n$. Note that

$$
L_{a_{1}} R_{b}=\sum_{j=1}^{m} R_{d_{j}} E R_{c_{j}} \in \mathcal{U} .
$$

We now return to the analytic setting. Let us first recall a theorem by Vala [26] which says that if $\mathcal{A}=\mathcal{B}(X)$, the algebra of all bounded linear operators on a Banach space $X$, then for any $a, b \in \mathcal{A}$ the operator $L_{a} R_{b}$ is compact on $\mathcal{A}$ if and only if $a$ and $b$ are compact operators on $X$.

Lemma 10.3. Let $\mathcal{A}$ be a primitive Banach algebra with $\operatorname{soc}(\mathcal{A}) \neq 0$ and let $\pi$ be the regular representation of $\mathcal{A}^{1}$ on the Banach space $X=\mathcal{A}$ e where $e$ is a minimal idempotent of $\mathcal{A}$. Further, let $E=\sum_{i=1}^{n} L_{a_{i}} R_{b_{i}}$ be a compact elementary operator on $\mathcal{A}$ and assume that $b_{1}$ does not lie in the linear span of $b_{2}, \ldots, b_{n}$. Then $\pi\left(a_{1}\right)$ is a compact operator on $X$. Moreover, if $\pi^{-1}$ is continuous then $a_{1}$ is a compact element.

Proof. Corollary 10.2 tells us that $L_{a_{1}} R_{b}$ is compact for some $b \neq 0$. Since $\mathcal{A}$ is a prime algebra, $e \mathcal{A} b \mathcal{A} e \neq 0$. Moreover, since $e \mathcal{A} e=\mathbb{C} e$ we have esbte $=e$ for some $s, t \in \mathcal{A}$. Therefore $L_{a_{1}} R_{e}=R_{t e}\left(L_{a_{1}} R_{b}\right) R_{e s}$ is a compact operator. Its restriction to $\mathcal{A} e=X$ is then also compact, meaning that $\pi\left(a_{1}\right)$ (defined by $\pi\left(a_{1}\right) x e=a_{1} x e$ ) is a compact operator on $X$. By 
Vala's theorem, $L_{\pi\left(a_{1}\right)} R_{\pi\left(a_{1}\right)}$ is a compact operator on $\mathcal{B}(X)$. Therefore the operator $L_{a_{1}} R_{a_{1}}=\pi^{-1} L_{\pi\left(a_{1}\right)} R_{\pi\left(a_{1}\right)} \pi$ is also compact provided that $\pi^{-1}$ is continuous.

In certain classes of algebras, $\pi^{-1}$ is automatically continuous. For example, this is true in the so-called ultraprime algebras introduced by Mathieu [17]: a normed algebra $\mathcal{A}$ is said to be ultraprime if there exists a constant $k_{\mathcal{A}}>0$ such that $\left\|L_{a} R_{b}\right\| \geq k_{\mathcal{A}}\|a\|\|b\|$ for all $a, b \in \mathcal{A}$. If $\mathcal{A}$ is ultraprime, then so is $\mathcal{A}^{1}$ [17, Proposition 3.7]. Since

$$
\begin{aligned}
\|\pi(a)\|\|e\| & \geq\|\pi(a)\| \sup _{\|x\|=1}\|x e\| \\
& \geq \sup _{\|x\|=1}\|\pi(a) x e\|=\sup _{\|x\|=1} \| \text { axe }\|=\| L_{a} R_{e} \|
\end{aligned}
$$

it follows that in an ultraprime algebra $\mathcal{A}$ we have $\|\pi(a)\| \geq k_{\mathcal{A}^{1}}\|a\|$ for every $a \in \mathcal{A}^{1}$, so that $\pi^{-1}$ is continuous. The class of ultraprime normed algebras contains prime $C^{*}$-algebras [18, Proposition 2.3], $\mathcal{B}(X)$ where $X$ is any normed space (one can check this easily and, moreover, $k_{\mathcal{B}(\mathcal{X})}=1$ ), and prime group algebras $l^{1}(G)$ where $G$ is a discrete group [27]. Further, ideals of ultraprime normed algebras are again ultraprime [17, Proposition 3.6]. On the other hand, quotients of ultraprime algebras by their closed ideals may not be ultraprime, and there exist primitive Banach algebras with nonzero socle that are not ultraprime [3].

A subset $\mathcal{M}$ of a Banach algebra $\mathcal{A}$ is called a bicompact subset of $\mathcal{A}$ if $L_{a} R_{b}$ is a compact operator for all $a, b \in \mathcal{M}$. For example, Vala's theorem shows that the set of compact operators on a Banach space $X$ is a bicompact subset of $\mathcal{B}(X)$. The next result generalizes this.

Corollary 10.4. If $\mathcal{A}$ is a semisimple ultraprime Banach algebra, then $\mathcal{A}_{\mathrm{c}}$ is a bicompact subset and a closed ideal of $\mathcal{A}$.

Proof. We may assume that $\mathcal{A}_{\mathrm{c}} \neq 0$. Thus $L_{a} R_{a}$ is a compact operator for some nonzero $a \in \mathcal{A}$, and $\operatorname{sos} \operatorname{soc}(\mathcal{A}) \neq 0$ by Theorem 9.2. A prime algebra with nonzero socle is primitive since the minimal left ideal $\mathcal{A} e$ is a faithful simple left $\mathcal{A}$-module for every minimal idempotent $e$. Let $\pi$ be as in Lemma 10.3. The lemma in particular implies that $\pi(a)$ is a compact operator if $a \in \mathcal{A}_{\mathrm{c}}$. So, if $a, b \in \mathcal{A}_{\mathrm{c}}$, then $\pi(a)$ and $\pi(b)$ are compact operators, and hence $L_{\pi(a)} R_{\pi(b)}$ is compact by Vala's theorem. Since $\pi^{-1}$ is continuous, it follows (as in the proof of Lemma 10.3) that $L_{a} R_{b}$ is compact on $\mathcal{A}$. Thus $\mathcal{A}_{\mathrm{c}}$ is a bicompact subset of $\mathcal{A}$. It is clearly a closed set, and in order to prove that it is an ideal we only have to show that it is closed under addition. Now, if $a, b \in \mathcal{A}_{\mathrm{c}}$, then

$$
L_{a+b} R_{a+b}=L_{a} R_{a}+L_{a} R_{b}+L_{b} R_{a}+L_{b} R_{b}
$$


is, as a sum of four compact operators (by what we just showed), a compact operator itself. Therefore $a+b \in \mathcal{A}_{\mathrm{c}}$.

The following is the main result of this section.

THEOREM 10.5. Let $\mathcal{A}$ be a semisimple ultraprime Banach algebra and let $E=\sum_{i=1}^{n} L_{a_{i}} R_{b_{i}}$ be an elementary operator on $\mathcal{A}$ such that $b_{1}$ does not lie in the linear span of $b_{2}, \ldots, b_{n}$. If $E$ is a compact operator then $a_{1}$ is a compact element.

Proof. Ultraprime normed algebras are centrally closed [17, Corollary 4.7]. Our assumption that $b_{1}$ does not lie in the linear span of $b_{2}, \ldots, b_{n}$ therefore in particular implies that $a_{1}=0$ if $E=0$ - this follows from Corollary 10.2 by choosing $\mathcal{U}=0$. We may therefore assume that $E \neq 0$. By Theorem $9.2, \mathcal{A}$ has a nonzero socle. Thererefore Lemma 10.3 implies that $a_{1}$ is a compact element.

Theorem 10.5 was known before in two special cases: when $\mathcal{A}=\mathcal{B}(X)[13$, Theorem 2] and when $\mathcal{A}$ is a prime $C^{*}$-algebra [19, Theorem 3.8]. It would be interesting to find other classes of prime Banach algebras for which the conclusion of this theorem holds (or does not hold). Let us point out that we used the ultraprimeness condition only to guarantee that $\mathcal{A}$ is centrally closed and $\pi^{-1}$ is continuous, so under certain technical conditions a more general result could be stated.

We remark that by making some rather obvious modifications in the proof of Theorem 10.5 one can prove an analogous statement: If $a_{1}$ does not lie in the linear span of $a_{2}, \ldots, a_{n}$ and $E$ is a compact operator, then $b_{1}$ is a compact element. Accordingly, if both sets $\left\{a_{1}, \ldots, a_{n}\right\}$ and $\left\{b_{1}, \ldots, b_{n}\right\}$ are linearly independent and the operator $\sum_{i=1}^{n} L_{a_{i}} R_{b_{i}}$ is compact, then all elements $a_{i}, b_{i}$ are compact.

Corollary 10.6. Let $\mathcal{A}$ be a semisimple ultraprime Banach algebra. If $E$ is a compact elementary operator on $\mathcal{A}$, then there exist compact elements $a_{1}, \ldots, a_{n}, b_{1}, \ldots, b_{n} \in \mathcal{A}^{1}$ such that $E=\sum_{i=1}^{n} L_{a_{i}} R_{b_{i}}$.

Proof. We may assume that $E \neq 0$. Therefore $E$ has length $n \geq 1$. If $E$ is represented as $E=\sum_{i=1}^{n} L_{a_{i}} R_{b_{i}}$, then clearly the sets $\left\{a_{1}, \ldots, a_{n}\right\}$ and $\left\{b_{1}, \ldots, b_{n}\right\}$ are linearly independent. As mentioned above, this implies that all $a_{i}, b_{i}$ are compact.

A result of this type also appears in [13] (for $\mathcal{B}(X)$ ) and in [19] (for prime $C^{*}$-algebras), and was also recently proved for general $C^{*}$-algebras [24].

Note that Corollaries 10.4 and 10.6 show that every compact elementary operator on a semisimple ultraprime Banach algebra is a sum of compact elementary operators of length 1 . 


\section{References}

[1] J. C. Alexander, Compact Banach algebras, Proc. London Math. Soc. 18 (1968), $1-18$.

[2] C. Apostol and L. Fialkow, Structural properties of elementary operators, Canad. J. Math. 38 (1986), 1485-1524.

[3] P. Ara and M. Mathieu, On ultraprime Banach algebras with non-zero socle, Proc. R. Ir. Acad. 91 Sect. A (1991), 89-98.

[4] B. A. Barnes, On the existence of minimal ideals in a Banach algebra, Trans. Amer. Math. Soc. 133 (1968), 511-517.

[5] K. I. Beidar, W. S. Martindale 3rd and A. V. Mikhalev, Rings with Generalized Identities, Dekker, 1996.

[6] M. Brešar, On the distance of the composition of two derivations to the generalized derivations, Glasgow Math. J. 33 (1991), 89-93.

[7] M. Brešar and D. Eremita, The lower socle and finite rank elementary operators, Comm. Algebra 31 (2003), 1485-1497.

[8] M. Brešar and W. S. Martindale 3rd, On the multiplication ring of a prime ring, Comm. Algebra 34 (2006), 2195-2203.

[9] M. Brešar and Yu. V. Turovskii, A note on compact semiderivations, in: Banach Center Publ. 67, Inst. Math., Polish Acad. Sci., 2005, 133-137.

[10] L. Dalla, S. Giotopoulos and N. Katseli, The socle and finite-dimensionality of a semiprime Banach algebra, Studia Math. 92 (1989), 201-204.

[11] H. V. Dedania, Compact elements in weighted discrete semigroup algebras, in: Banach Algebras 97, de Gruyter, Berlin, 1998, 119-134.

[12] T. S. Erickson, W. S. Martindale 3rd and J. M. Osborn, Prime nonassociative algebras, Pacific J. Math. 60 (1975), 49-63.

[13] C. K. Fong and A. Sourour, On the operator identity $\sum A_{k} X B_{k} \equiv 0$, Canad. J. Math. 31 (1979), 845-857.

[14] T.-K. Lee and T.-L. Wong, Semiprime algebras with finiteness conditions, Comm. Algebra 31 (2003), 1823-1835.

[15] R. J. Loy and G. A. Willis, The approximation property and nilpotent ideals in amenable Banach algebras, Bull. Austral. Math. Soc. 49 (1994), 341-346.

[16] B. Magajna, A system of operator equations, Canad. Math. Bull. 30 (1987), 200-209.

[17] M. Mathieu, Rings of quotients of ultraprime Banach algebras. With applications to elementary operators, Proc. Centre Math. Anal. Austral. Nat. Univ. 21 (1989), $297-317$.

[18] - Elementary operators on prime $C^{*}$-algebras I, Math. Ann. 284 (1989), 223-244.

[19] - Elementary operators on prime $C^{*}$-algebras II, Glasgow Math. J. 30 (1988), $275-284$.

[20] L. Rowen, Ring Theory, Academic Press, 1991.

[21] V.S. Shulman and Yu. V. Turovskii, On the Kleinecke-Shirokov theorem for families of derivations, Studia Math. 149 (2002), 185-190.

[22] - - - Topological radicals, II, preprint.

[23] M. R. F. Smyth, On problems of Olubummo and Alexander, Proc. R. Ir. Acad. 80A (1980), 69-74.

[24] R. M. Timoney, Some formulae for norms of elementary operators, J. Operator Theory, to appear.

[25] Yu. V. Turovskii and V. S. Shulman, Radicals in Banach algebras, and some problems of theory of radical Banach algebras, Funktsional. Anal. i Prilozhen. 35 (2001), no. 4, 88-91 (in Russian). 
[26] K. Vala, On compact sets of compact operators, Ann. Acad. Sci. Fenn. Ser. A I 351 (1964).

[27] G. A. Willis, Ultraprime group algebras, Proc. Centre Math. Anal. Austral. Nat. Univ. 21 (1989), 345-349.

Department of Mathematics

University of Maribor

PEF, Koroška 160

2000 Maribor, Slovenia

E-mail: bresar@uni-mb.si
Institute of Mathematics and Mechanics National Academy of Sciences of Azerbaijan

F. Agaev St. 9

Baku AZ1141, Azerbaijan

E-mail: yuri.turovskii@gmail.com

yuri@sinam.net

Received September 26, 2005

Revised version April 6, 2006 